\title{
Segmentation and Measurement of the Cortex from 3D MR Images
}

\author{
Xiaolan Zeng ${ }^{1}$, Lawrence H. Staib ${ }^{1}$, Robert T. Schultz ${ }^{2}$, and James S. Duncan ${ }^{1}$ \\ 1 Departments of Electrical Engineering and Diagnostic Radiology, \\ 2 Child Study Center, Yale University, New Haven, CT, 06520-8042
}

\begin{abstract}
The cortex is the outermost thin layer of gray matter in the brain; geometric measurement of the cortex helps in understanding brain anatomy and function. In the quantitative analysis of the cortex from MR images, extracting the structure and obtaining a representation for various measurements are key steps. While manual segmentation is tedious and labor intensive, automatic, reliable and efficient segmentation and measurement of the cortex remain challenging problems due to its convoluted nature. A new approach of coupled surfaces propagation using level set methods is presented here for the problem of the segmentation and measurement of the cortex. Our method is motivated by the nearly constant thickness of the cortical mantle and takes this tight coupling as an important constraint. By evolving two embedded surfaces simultaneously, each driven by its own image-derived information while maintaining the coupling, a final representation of the cortical bounding surfaces and an automatic segmentation of the cortex are achieved. Characteristics of the cortex such as cortical surface area, surface curvature and thickness are then evaluated. The level set implementation of surface propagation offers the advantage of easy initialization, computational efficiency and the ability to capture deep folds of the sulci. Results and validation from various experiments on simulated and real 3D MR images are provided.
\end{abstract}

\section{Introduction}

A significant amount of the recent anatomical MRI studies on the human brain have been focused on the cerebral cortex. The cerebral cortex is the outermost layer of gray matter in the brain. It is composed of columns of neurons, aligned perpendicularly to the cortical surface, that serve as basic units of information processing in the brain. Cortical surface area is likely to be proportional to column number and therefore surface area should be related to functional capacities. In addition, regional cortical thickness and gray matter volume may relate to functional capacities, and alteration in each of these features has been suspected in specific neuropsychiatric disorders([17]). In the quantitative analysis of these features of the cortex, segmentation is the first step.

The cerebral cortex is characterized by its convoluted surface. Due to this convoluted nature, the segmentation of the cortex must be considered in 3D. For example, although the cerebral cortical layer is about $3 \mathrm{~mm}$ thick([1]), an oblique $2 \mathrm{D}$ slice that happens to be approximately parallel to a particular sulcus will give the appearance of a much thicker structure. Only by going through 
the neighboring slices can we get complete information to perform segmentation. Slice by slice manual tracing of the cortex is extremely tedious and labor intensive, hence automatic, reliable and relatively efficient segmentation which enables automated measurement is a highly desirable goal.

\subsection{Related work}

There has been a large amount of work devoted to automatic 3D segmentation. One type of approach uses region-based methods, which exploit homogeneity in images. Following the work of Geman \& Geman[7], Markov Random Field(MRF)-based methods have been widely used. For example, a multispectral voxel classification method was used in conjunction with connectivity to segment the brain into different tissue types from 3D MR images[2]. Material mixture models were also used for the segmentation problem. Region-based methods typically require further processing to group segmented parts into coherent structure(s). Moreover, quantitative measurement of features other than volume does not follow immediately.

A typical alternative strategy is boundary finding, of which active contour methods are of special note. They rely mainly on gradient features for segmentation of structures. For instance, a 3D deformable surface model using the finiteelement method was used to segment 3D images[3]. One concern regarding this type of method is that close initialization has to be provided in order to achieve good final results. Also, the need to override the local smoothness constraint to allow for significant protrusions (which is highly desirable in order to capture the sulcal folds) remains a problem.

An iterative algorithm was presented by MacDonald et al. for simultaneous deformation of multiple surfaces to segment MRI data with inter-surface constraints and self-intersection avoidance, where surface deformation was formulated as a cost function minimization problem[13]. This method was applied to 3D MR brain data to extract surface models for the skull and the cortical surface. This approach takes advantage of the information of the interrelation between the surfaces of interest. However, drawbacks lie in its extremely high computational expense, and the difficulty of tuning the weighting factors in the cost function due to the complexity of the problem.

Teo et al.[19] used a system that exploited knowledge of cortical anatomy, in which white matter and CSF regions were first segmented, then the connectivity of the white matter was verified in regions of interest. Finally a connected representation of the gray matter was created by a constrained growing-out from the white matter boundary. The focus of this work was to create a representation of cortical gray matter for functional MRI visualization.

Davatzikos et al. [4] introduced the concept of a ribbon for modeling the outer cortex in cross-sectional brain images and then extended the model into 3D[5]. A deformable surface algorithm was constructed to find the central layer of the cortex. Based on this parameterization, the cortical structure was characterized through its depth map and curvature map. This method explicitly used the structural information of the cortex. However, close initialization and significant human interaction are needed to force the ribbon into sulcal folds. 


\section{Our Approach}

The cortical layer to be recovered has an nearly constant thickness and is bounded by two surfaces: the CSF/gray matter boundary and gray/white matter boundary. Across each surface, there is a local difference in the gray level values, while in between the two surfaces there is a homogeneity of certain voxel statistics. For our purposes, the cortical layer is defined completely by its bounding surfaces and the homogeneity in between. Following our earlier work[20], we propose a new approach of coupled surfaces propagation via level set methods for the segmentation and measurement of the cortex. By evolving two embedded surfaces simultaneously, each driven by its own image-based information while maintaining the coupling, we are able to achieve an automatic and robust segmentation of the cortex, and simultaneously obtain a representation of the inner and outer cortical surfaces from which surface area can be calculated. Furthermore, curvature and thickness maps are easily obtained from this coupled level set formulation.

\subsection{Image information derivation}

In order to capture the notion of homogeneity inside the cortical layer, we have designed an image gray level-based local operator to obtain the likelihood of each voxel lying on the outer and inner cortical surfaces respectively, instead of using the gradient information alone. This model can potentially be extended to make use of a vector of registered parametric images (such as T1, T2 and PD MR images) or images from different modalities.

At each voxel site $s$, a small neighborhood around $s$ is drawn(see Figure 2). Now given a possible boundary with normal direction $\theta$, dividing the neighborhood into parts $R 1$ and $R 2$, the probability that $s$ lies on the boundary between tissue $A$ and tissue $B$ is:

$$
p_{A B}(\boldsymbol{\theta})=p(R 1 \in \text { Tissue } A) \cdot p(R 2 \in \text { TissueB })
$$

Given an estimation $\boldsymbol{\theta}^{*}$ of $\boldsymbol{\theta}$, we can use $p_{A B}\left(\boldsymbol{\theta}^{*}\right)$ as a measure of the likelihood that $s$ lies on the boundary between tissue $A$ and tissue $B$.

One way of estimating $\boldsymbol{\theta}^{*}$ is to first generate the vector $P=\left[p\left(\boldsymbol{\theta}_{1}\right), p\left(\boldsymbol{\theta}_{2}\right), \ldots\right.$, $\left.p\left(\theta_{k}\right)\right]^{T}$, and $\boldsymbol{\theta}^{*}$ is then the one which corresponds to the element of largest magnitude in vector $P$. Here we make the assumption of a single parametric image $X$, in which voxels belonging to tissue $A$ are independently drawn from a Gaussian distribution $G\left(\mu_{A}, \sigma_{A}\right)$, and voxels belonging to tissue $B$ are independently drawn from $G\left(\mu_{B}, \sigma_{B}\right)$. We have

$$
p_{A B}(\boldsymbol{\theta})=\prod_{r \in R 1} \frac{1}{\sqrt{2 \pi} \sigma_{A}} e^{-\frac{\left(X_{r}-\mu_{A}\right)^{2}}{\sigma_{A}^{2}}} \cdot \prod_{t \in R 2} \frac{1}{\sqrt{2 \pi} \sigma_{B}} e^{-\frac{\left(\boldsymbol{X}_{t}-\mu_{B}\right)^{2}}{\sigma_{B}^{2}}}
$$

In Figure 1, we show an example of the result from our local operator. The local operator was applied to images after we reduced the effects of MR inhomogeneity by correcting using a simple fixed map. The map was determined manually by sampling tissue types throughout the field to decide the average inhomogeneity. Note that more complicated MR image models $([7],[11],[10])$ can be used to calculate $p(\theta)$. 
a)
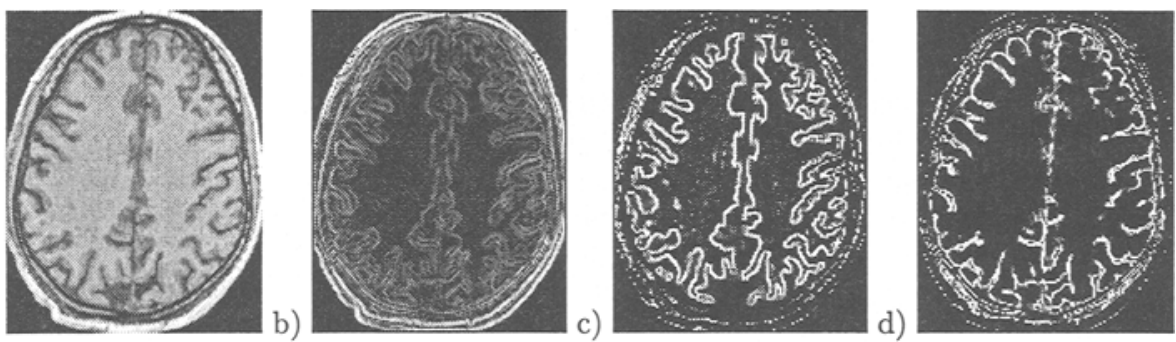

Fig. 1. Results from our local operator compared to image gradient. (a): axial slice from the original 3D brain images; (b): result from gradient operator; (c): result from our local operator $p_{B C}\left(\boldsymbol{\theta}^{*}\right), \mathrm{B}=$ gray matter, $\mathrm{C}=$ white matter; $(\mathrm{d}) p_{A B}\left(\boldsymbol{\theta}^{*}\right), \mathrm{A}=\mathrm{CSF}$, $\mathrm{B}=$ gray matter.

\subsection{Level set method}

Level set methods $([18],[14],[15])$ are powerful numerical techniques for analyzing and computing interface motion. The essential idea is to represent the surface (in our case) of interest as a front $\gamma(t)$, and embed this propagating front as the zero level set of a higher dimensional function $\Psi$ defined by $\Psi(x, t)=d$, where $d$ is the signed distance from position $x$ to $\gamma(t)$. An Eulerian formulation is produced for the motion of this surface propagating along its normal direction with speed $F$, where $F$ can be a function of the surface characteristics (such as the curvature, normal direction etc.) and the image characteristics (e.g. the gray level, gradient etc.) The equation of the evolution of $\Psi$, inside which our surface is embedded as the zero level set is then given by $\Psi_{t}+F|\nabla \Psi|=0$.

The major advantages of using this method over other active contour strategies include the following. First, the evolving level function $\Psi(x, t)$ remains a function; however the propagating front $\gamma(t)$ may change topology, break, merge (this allows the flexibility of the initialization in image segmentation) and form sharp corners as $\Psi$ evolves. Second, the intrinsic geometric properties of the front may be easily determined from $\Psi$. For example, at any point of the front, the normal vector is given by $n=\nabla \Psi$.

\subsection{Coupled surfaces propagation, speed term design}

In solving the problem of segmenting the cortex, we consider two moving interfaces describing the inner and outer cortical bounding surfaces respectively. Starting from inside the inner cortical surface (i.e. inside the white matter), with an offset in between, the interfaces propagate along the outward normal direction and stop at the desired place, while maintaining the distance between them.

Embedding each surface as the zero level set in its own level function, we have two equations:

$$
\begin{aligned}
\Psi_{\text {in }}+F_{\text {in }}\left|\nabla \Psi_{\text {in }}\right| & =0 \\
\Psi_{\text {out }}+F_{\text {out }}\left|\nabla \Psi_{\text {out }}\right| & =0
\end{aligned}
$$

where $F_{\text {in }}$ and $F_{\text {out }}$ are functions of the surface normal direction, image-derived information and distance between the two surfaces. The coupling is embedded 
in the design of $F_{\text {in }}$ and $F_{\text {out }}$. At places where the distance between the two surfaces is within the normal range, the two surfaces propagate according to the image-based information. Where the distance between the two surfaces is out of the normal range, the distance imposes a constraint on the propagation of the surfaces.

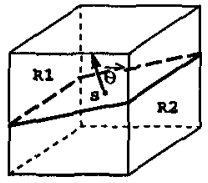

Fig. 2. A local operator to derive image information.

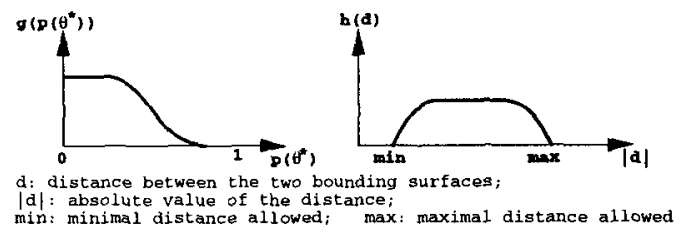

Fig. 3. Functions $g$ and $h$ used in speed term design.

The motivation behind using the coupling constraint between the two surfaces is to make the image information on the partner surface available to the other, thus improving the segmentation result. For example, due to volume averaging, in some regions of the image, the inner cortical boundary may not be well defined while the CSF appears clearly. A single surface approach might result in the inner cortical surface collapsing into CSF. However with the coupling constraint, a minimum distance from the inner cortical surface to CSF is maintained, thus preventing the inner surface from going into CSF. There are also cases near structures such as eye sockets where the CSF can not be observed. While a single surface will grow into these foreign structures, the coupled surfaces approach stops the propagation of the outer cortical surface using the information from the inner cortical surface. (see Figure 9)

With the level set implementation, we have a natural way to establish a correspondence between the points on the two evolving surfaces through distance, which is evaluated without extra computational expense. Recall that the value of the level function of a front at any point is simply the distance from this point to the current front, which is calculated as the shortest distance from this point to all the points on the front[18]. In our case of two moving surfaces, for any point on the inner moving surface, the distance to the outer moving surface is the value $\Psi_{\text {out }}$ at this point, and vice versa for the point on the outer moving surface. Hence, we write

$$
\begin{aligned}
F_{\text {in }} & =g\left(p_{B C}\left(\boldsymbol{\theta}^{*}\right)\right) h\left(\Psi_{\text {out }}\right) \\
F_{\text {out }} & =g\left(p_{A B}\left(\boldsymbol{\theta}^{*}\right)\right) h\left(\Psi_{\text {in }}\right)
\end{aligned}
$$

where $g$ and $h$ are the functions as shown in Figure 3, and $A, B, C$ denote CSF, gray matter and white matter respectively.

Function $g$ maps larger likelihood to slower speed, i.e., as the likelihood gets larger, $g$ tends to zero, while as the likelihood gets to near zero, $g$ tends to a constant. Function $h$ penalizes the distance off the normal range. As the distance 
goes out of normal range, $h$ goes to zero. Thus, each surface moves with constant speed along the normal direction and slows down when either the image-based information becomes strong or the distance to the other surface moves away from the normal range. Each surface finally stops when the image-derived information is strong enough or the distance to the other surface is out of the normal range. The speed term only has meaning on the front, i.e. the zero level set. It is then extended from the zero level set to the whole image grid[18]. For computational efficiency, the algorithm is implemented using a narrow band method, which modifies the level set method so that only points close to the current propagating fronts are affected [20].

\subsection{Measurement}

With coupled surfaces propagation via the level set method, it is easy to perform various measurements on the cortical layer with little extra computational expense. Whole brain volume, cortical gray matter volume, white matter volume, cortical surface area, cortical surface shape and cortical thickness maps are among the features most interesting in the study of brain structure and function. Different combinations of the above measurements may help in determining the pathobiology of various neuropsychiatric disorders. We now discuss one by one the above measurements from our coupled surfaces formulation.

Volume With the signed distance function $\Psi$, the level set formulation keeps track of the inside and outside of the current moving front. Once the evolution of the coupled surfaces is completed, the cortical gray matter voxels are those that lie inside the outer cortical surface while outside the inner cortical surface. In the same fashion, non-brain tissue voxels will be the ones that are outside the outer cortical surface, and voxels of white matter will lie inside the inner cortical surface except for subcortical gray matter and ventricles. Because the signed distance based measures are more accurate than the voxel size based measures, we can obtain a partial volume segmentation on the data set, instead of a binary segmentation. In other words, if the distance from a voxel to the measured cortical boundary is less than the voxel size in width, the voxel is considered to contain multiple tissue types.

Surface area A marching cubes algorithm[12] is performed on the signed distance functions, $\Psi_{i n}$ and $\Psi_{\text {out }}$, to extract the embedded zero level sets, in this case the inner and outer cortical surfaces, when the evolution is completed. The surfaces are realized using a triangular representation. Surface area is then calculated as the sum of the areas of the triangles.

Surface curvature and shape index As discussed above, one advantage of the level set implementation is that geometric properties of the propagation front are easily calculated[18]. In our case of surfaces propagating in 3D space, there are many choices for the curvature of the front (for formal definitions of the curvatures, refer to [6]), including mean curvature, $\kappa_{M}$, and Gaussian curvature, $\kappa_{G}$. Both may be conveniently expressed in terms of the level set function $\Psi$ : $\kappa_{M}=\left\{\sum_{(i, j, k) \in C}\left(\left(\Psi_{i i}+\Psi_{j j}\right) \Psi_{k}^{2}-2 \Psi_{i} \Psi_{j} \Psi_{i j}\right)\right\} /\left\{2\left(\Psi_{x}^{2}+\Psi_{y}^{2}+\Psi_{z}^{2}\right)^{3 / 2}\right\}$, and $\kappa_{G}=$ 
$\left\{\sum_{(i, j, k) \in C}\left(\Psi_{i}^{2}\left(\Psi_{j j} \Psi_{k k}-\Psi_{j k}^{2}\right)+2 \Psi_{i} \Psi_{j}\left(\Psi_{i k} \Psi_{j k}-\Psi_{i j} \Psi_{k k}\right)\right)\right\} /\left(\Psi_{x}^{2}+\Psi_{y}^{2}+\Psi_{z}^{2}\right)^{2}$, where $C=\{(x, y, z),(y, z, x),(z, x, y)\}$ is the set of circular shifts of $(x, y, z)$.

The maximum principle curvature, $\kappa_{1}$, and the minimum principle curvature, $\kappa_{2}$, are related to Gaussian and mean curvatures through the following formulas: $\kappa_{1}=\kappa_{M}+\sqrt{\kappa_{M}^{2}-\kappa_{G}}$, and $\kappa_{2}=\kappa_{M}-\sqrt{\kappa_{M}^{2}-\kappa_{G}}$. We adopt the classification of surfaces by Koenderink $[8]$ using the numerical relationship between the two principal curvatures. A shape index function is defined as $s i=\frac{2}{\pi} \arctan \left(\left(\kappa_{1}+\kappa_{2}\right) /\left(\kappa_{1}-\kappa_{2}\right)\right)$, which classifies the surfaces into nine types as show in Figure 7 . With the shape index, gyri (mostly ridges) and sulci (mostly ruts) are automatically identified. Further potential uses of the shape index includes the definition of an atrophy index (sulci widen with age).

Thickness map As discussed above, the value of the level function of a front at any point is the distance from this point to the current front. Also recall that the inner and outer cortical surfaces are the zero level sets of $\Psi_{\text {in }}$ and $\Psi_{\text {out }}$. Thus, for any point on the outer cortical surface, the absolute value of $\Psi_{i n}$ at the point is simply the distance from the point to the inner cortical surface. Using this measure, we obtain a thickness map between the inner and outer cortical surfaces, which can be used to study the normal thickness variations across different regions of the brain, and also the abnormalities in brain structures.

\section{Experimental Result}

\subsection{Result on simulated MR data with ground truth}

We first present our segmentation results on the simulated MR brain images provided by the McConnell Brain Imaging Center at the Montreal Neurological Institute(http://www.bic.mni.mcgill.ca/). The images are generated using an MRI simulator [9], that allows users to independently control various acquisition parameters and obtain realistic MR images. The ground truth of the phantom is provided in the form of membership functions of each voxel belonging to different tissue types, such as the skull, CSF, gray matter and white matter.

The simulated data we tested our algorithm on were $\mathrm{T} 1$ images of a normal brain, with the following parameters: voxel size $=1 \mathrm{~mm}^{3}$, noise $=3 \%$, intensity non-uniformity $=0 \%$. Starting from the unedited images, no further user interaction is needed after the specification of several pairs of concentric spheres as initialization. The spheres grow out and automatically lock onto the inner and outer cortical surfaces. As long as the spheres are placed inside the white matter, the algorithm is robust to starting position. Measurement of the volume is then done as described in the previous section; we use a binary segmentation in this experiment.

To evaluate the segmentation result we apply several measures defined as follows. For any tissue type $T$ in the region of interest, we denote the voxels of tissue type $T$ recovered from our $3 \mathrm{D}$ algorithm as $V_{a}$ and the voxels that are mostly of tissue type $T$ according to the phantom(i.e. the value of tissue $T$ membership function is greater than 0.5) as $V_{e}$. We denote the overlap of $V_{a}$ and $V_{e}$ as $V_{a e}$, and the part that is in $V_{a}$ but not in $V_{e}$ as $V_{a e^{\prime}}$. A true positive(TP) rate is then defined to be the size of $V_{a e}$ relative to the size of $V_{e}$, while the false positive $(F P)$ rate is defined to be the ratio of the size of $V_{a e^{\prime}}$ to the size of $V_{e}$. 
We also define the volume ratio to be the volume of all the voxels segmented as of tissue type $T$ by our algorithm to the total partial volume of tissue type $T$ specified by the phantom(partial volume voxels contribute in only part of the voxel size).

\begin{tabular}{|c|c|c|c|c|}
\hline$\%$ & whole brain & cortical gray matter1 & cortical gray matter2 & white matter \\
\hline TP rate & 92.3 & 93.5 & 92.4 & 92.4 \\
\hline FP rate & 2.0 & 5.9 & 6.0 & 3.3 \\
\hline volume ratio & 96.3 & 103.9 & 102.7 & 98.1 \\
\hline
\end{tabular}

Table 1. Comparison of our volume measurements with the phantom ground truth. whole brain: total brain tissue(white+gray matter); cortical gray matter1: on the frontal 49 Coronal slices; cortical gray matter2: on the top 56 Axial slices;

Table 1 shows our measurement results over 4 types: total brain tissue (including white and gray matter), cortical gray matter in selected slices and the white matter. Since the algorithm is designed specifically for the nearly constant thickness of the cerebral cortex, it recovers only part of the gray matter in the brain stem and the cerebellum where the constant thickness constraint is not satisfied. These regions account for most of the errors in the TP rate and volume ratio for the whole brain tissue. Since no ground truth regarding the structural information is provided for the phantom, we compare the cortical gray matter volume on selected slices: frontal 49 Coronal slices and top 56 Axial slices(where there are only white matter and cortical gray matter). The average errors of the $T P$ and $F P$ rates are around $6 \%$ to $7 \%$, and the volume ratio error is within $4 \%$. For the white matter, the errors for the TP, FP rates and volume ratio are also low. These results show that our algorithm performs well in isolating the brain from non-brain tissues and in segmenting the cortex from simulated data.
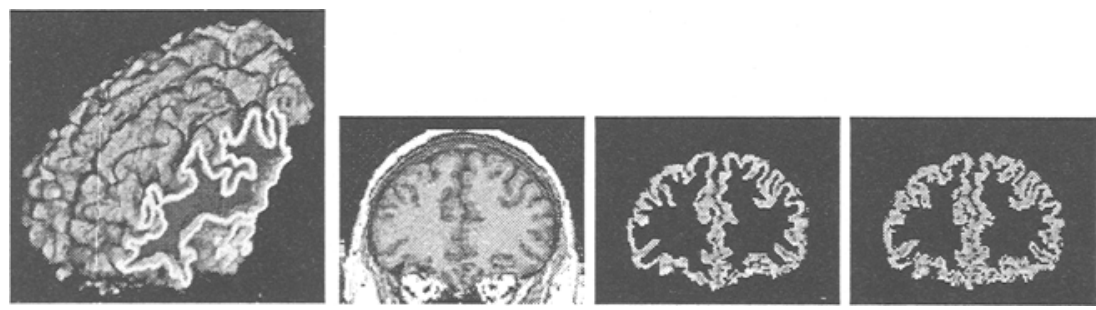

Fig. 4. 3D volume rendering of the cortex from our $3 \mathrm{D}$ algorithm with oblique cutting planes. The convoluted thin bright ribbon is the cortical gray matter captured on the cutting plane.
Fig. 5. Coronal slices from 3D images; left: original image; middle: cortical gray matter from manual tracing; right: cortical gray matter from our $3 \mathrm{D}$ algorithm.

\subsection{Result on real MR data}

We then tested our algorithm on frontal lobes of 7 high resolution MRI data sets(SPGR, 2NEX, $1.2 \times 1.2 \times 1.2 \mathrm{~mm}^{3}$ voxels) from a randomly chosen subset of 
young adult autistic and control subjects from our ongoing studies, to measure frontal lobe volume, gray and white matter volume, cortical surface area, surface curvature and cortical thickness. After preprocessing to reduce the effects of the MR bias field inhomogeneity using a simple standard nonlinear map (which is also a step before expert manual tracing), we used a single plane to isolate the part of the $3 \mathrm{D}$ image that contained the frontal lobe, and then ran the coupled surfaces algorithm on this part of the image to isolate the brain tissue and segment the cortex(see Figure 6). The frontal lobe was then defined independently in the left and right hemispheres as all tissue anterior to the central sulcus. Sub-cortical nuclei were excluded from our definition of the frontal volume.

Figure 4 shows a $3 \mathrm{D}$ volume rendering of the cortical gray matter of a frontal lobe resulted from our algorithm. In Figure 5, 2D Coronal slices of the same result are shown. Over the 7 frontal lobes, the TP and FP rates (compared to manual tracing) of the whole frontal lobe averaged $94.1 \%$ and $2.1 \%$ respectively, which demonstrated that our algorithm nicely isolated the brain tissue from the non-brain tissue. The average $T P$ and $F P$ rate for the gray matter (measured on 2 slices) in the frontal lobe were $86.7 \%$ and $20.8 \%$. As we see in Figure 5 , the expert tracing tended to be more aggressive in defining the gray/white matter boundary, which resulted in the relatively larger value of the $F P$ rate. As a second way to analyze the utility of the volume measurements from our algorithm, we compute the reliability statistics on the volume measurements[16]. There was strong agreement between the algorithm and the expert on the volume of the frontal lobe (Pearson $r=.991$; intraclass correlation coefficient $[\mathrm{ICC}]=.901$ ). The algorithm systematically estimated the frontal lobe volume to be less than the expert tracer (mean difference $=4 \%$ ), and this accounts for the lower ICC than Pearson coefficient. Similarly, for gray matter volume of the frontal lobe there was also good agreement (Pearson $r=.96$ ).

The outer and inner cortical surfaces captured by the algorithm were masked using the frontal lobe volume information to retain only the parts of the surfaces that are from the frontal lobe as defined above. The average outer and inner cortical surface areas over the 7 frontal lobes are $48800 \mathrm{~mm}^{2}$ and $63200 \mathrm{~mm}^{2}$ respectively. Figure 7 shows the outer and inner cortical surfaces of a frontal lobe colored with their shape indices. As we can see, most parts of the gyri are automatically identified as ridge while most parts of the sulci are identified as rut, which coincides with our knowledge of the cortical structure.

The average cortical thickness of the 7 frontal lobes ranged from $3.2 \mathrm{~mm}$ to $3.6 \mathrm{~mm}$, which matches data from postmortem studies([1]). The cortical thickness map displayed in Figure 8 also shows an interesting pattern of information, with gyral crowns represented as thicker than sulcal troughs. This also is in good agreement with postmortem data $([1])$. In addition, the primary motor strip and immediately adjacent premotor areas generally appear to contain thicker cortical gray matter than middle frontal areas, and this too is in agreement with known regional variations in cortical thickness ([17],[1]).

Assessing the relationships between surface areas and volumes, we see that the total inner surface area of the frontal lobe correlated quite strongly with the total outer surface area $(r=.82)$. Inner and outer surface areas also predicted total gray matter volume ( $r$ 's $=.76$ and .85 , respectively). The imperfect relationships suggest some measurement error, but also the possibility that surface area adds unique information beyond that provided by gray matter volume. 

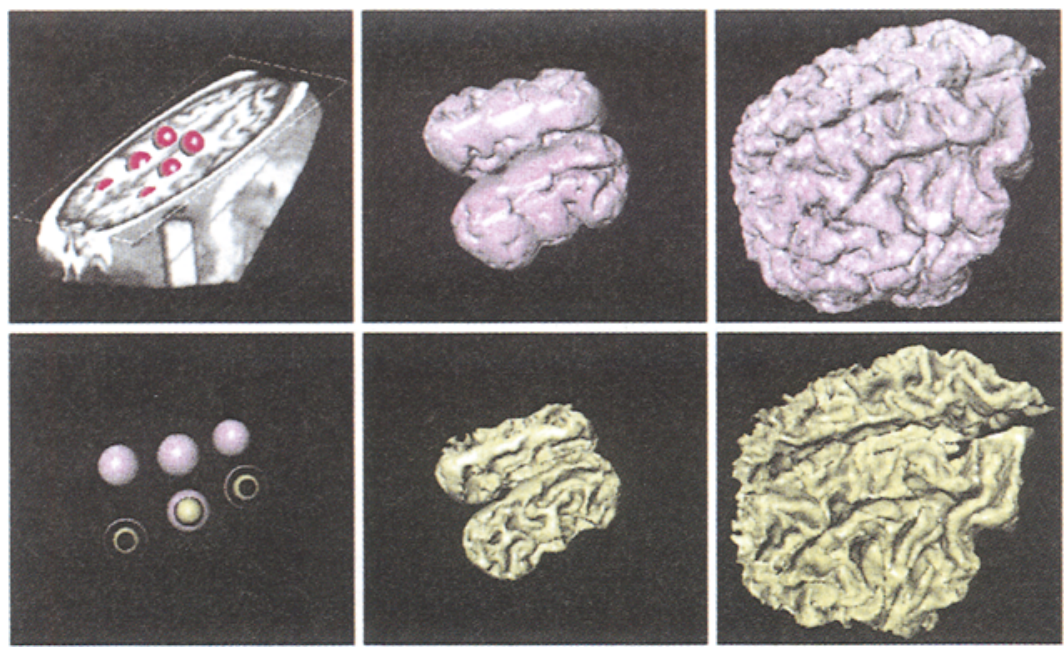

Fig. 6. Propagation of the outer(pink) and inner(yellow) bounding surfaces. left: pairs of concentric spheres(only the outer ones are shown at the top) as initialization in unedited 3D MR brain images(frontal part); middle: intermediate step; right: final result of the outer and inner cortical surfaces of the frontal lobe.

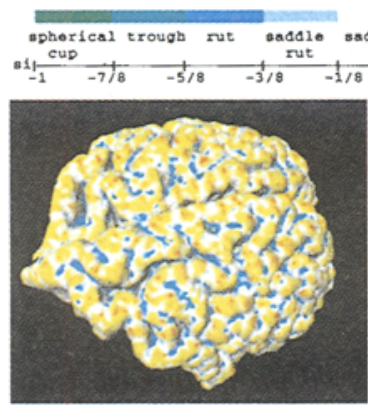

Fig. 7. The outer and inner cortical surfaces of a frontal lobe colored with the specified spectrum representing shape index(si).
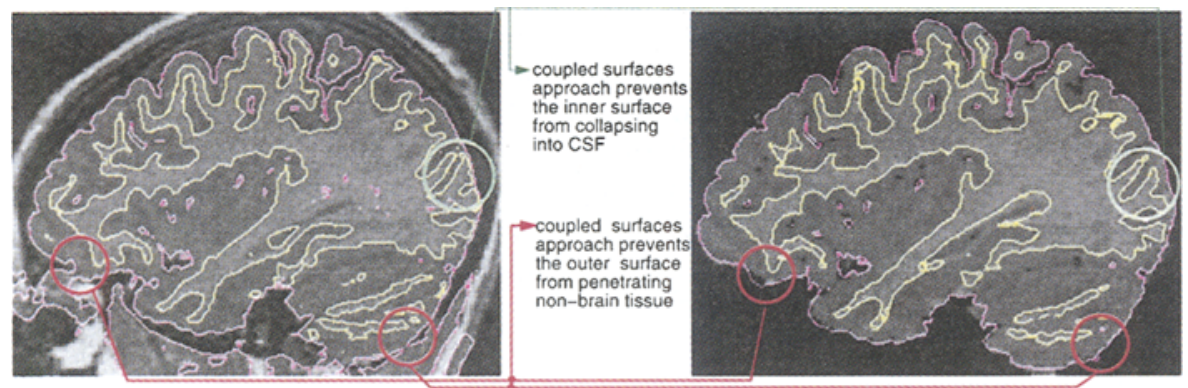

Fig. 9. Single vs. coupled surfaces approach. left: surfaces resulting from single surface approach on a sagittal slice of original image (finding the inner and outer surfaces separately); right: surfaces resulting from the coupled surfaces approach on a sagittal slice of the expert tracing result. 
Similarly, average gray matter thickness of the frontal lobe was assessed. These measurements were not significantly correlated with gray matter volume $(\mathrm{r}=$ .25 ) or surface areas (inner $=-.38$; outer $=-.10$ ), suggesting that thickness is nearly a constant and provides unique biological information.

\subsection{User interaction and speed issue}

Minimum user interaction and computational efficiency have always been two important issues in the problem of segmenting and measuring the cortex. For an expert to manually isolate the non-brain tissue alone (using computer-assisted manual tracing slice by slice) takes 3 to 4 hours. The manual tracing of cortical gray matter is even more time consuming. The multiple surface method of Macdonald et al.[13], could only be computed at medium resolution due to the computational expense. Davatzikos and Bryan[5] report that the "ribbon" algorithm was a fairly computationally demanding iterative procedure; while manual placement of the initial cortical surface and a multi-scale formulation could decrease the computational load (no processing time was given). The initialization for our algorithm only requires the user to specify several pairs of concentric spheres in the white matter, which can be done with several mouse clicks. It should be emphasized that neither the number nor the placement of the spheres affects the accuracy or the reproducibility of the final result. For a $3 \mathrm{D}$ image $\left(1.2 \times 1.2 \times 1.2 \mathrm{~mm}^{3}\right.$ in voxel size $)$ of the whole brain, the algorithm runs in about 1 hour on a R10000 SGI Indigo2 machine. Skull-stripping, segmentation and measurement of the cortex are done simultaneously. Comparatively, to our knowledge our algorithm outperforms other related techniques with respect to user interaction and computational efficiency.

\section{Summary and Future Directions}

In this paper, we presented a new approach to the segmentation and measurement of cortical structure which is of great interest in the study of the structural and functional characteristics of the brain. Motivated by the fact that the cortex has a nearly constant thickness, we model the cortex as a volumetric layer, which is completely defined by the two bounding surfaces and the homogeneity in between. Starting from easily initialized spheres, and driven by image-derived information, two interfaces evolve out to capture the inner and outer cortical boundaries, thereby segmenting out the cortical gray matter from the white matter, as well as isolating the brain tissue from the non-brain tissue. Cortical gray matter volume and cortical surface area (both inner and outer) are then measured. Due to the coupled level set implementation, the cortical surface curvature and cortical thickness map are also easily obtained. As seen from various experiments, our algorithm is automatic, accurate, robust and relatively computationally efficient.

Future directions of our research include the following: volume measurement on the sub-voxel level, possible use of a vector image data set, and testing on image data of abnormal brains.

Acknowledgment This work was supported in part by NIH grant NINDS R01 NS35193, NSF grant IRI-9530768, and NIH grant NICHD 5 P01 HDIDC 35482 . 


\section{References}

1. S.M.Blinkov and I.I.Glezer. The Human Brain In Figures and Tables. A Quantitative Handbook. Chapter X, pp182. Basic Books,Inc., Plenum Press, 1968.

2. H.E.Cline, W.E.Lorensen, R. Kikinis, and F.Jolesz. Three-dimensional segmentation of MR images of the head using probability and connectivity. J. Comput. Assist. Tomogr., 14(6):1037-1045, Nov./Dec. 1990.

3. L.D.Cohen and I.Cohen. Finite-Element methods for active contour models and balloons for 2-D and 3-D images. IEEE Trans. Pattern Anal. Machine Intell., 15(11):1131-1147, Nov, 1993.

4. C.A.Davatzikos and J.Prince. An active contour model for mapping the cortex. IEEE Trans. Med. Imag., 14(1):65-80, March, 1995.

5. C.Davatzikos and R.N.Bryan. Using a deformable surface model to obtain a shape representation of cortex. IEEE Trans. Med. Imag., 15(6):785-795, 1996.

6. M.P.DoCarmo. Differential Geometry of Curves and Surfaces. Prentice-Hall, New Jersey, 1976.

7. D.Geman and S.Geman. Stochastic relaxation, Gibbs distribution and Bayesian restoration of images. IEEE Trans. Pattern Anal. Machine Intell., 6:721-741, 1984.

8. J.J.Koenderink and A.J.van Doorn. Surface shape and curvature scale. Image and Vision Computing, 10(8):557-565, 1992.

9. R.K.-S.Kwan, A.C.Evans and G.B.Pike, An Extensible MRI Simulator for PostProcessing Evaluation, Visualization in Biomedical Computing vol. 1131, pp 135140, Springer-Verlag, 1996.

10. S.Lakshmanan and H.Derin. Simultaneous Parameter Estimation and Segmentation of Gibbs Random Fields Using Simulated Annealing. IEEE Trans. Pattern Anal. and Machine Intell., 11(8):799-810, 1989.

11. R.Leahy, T.Hebert and R.Lee. Applications of Markov Random Fields in medical imaging. Information Processing in Medical Imaging. pp:1-14. Wiley-Liss Inc, 1991.

12. W.Lorenson and H.Cline. Marching Cubes: A High Resolution 3D Surface Construction Algorithm. Proc. SIGGRAPH, 21(4):163-169, July 1987.

13. D.MacDonald, D. Avis and A.E.Evans. Multiple Surface Identification and Matching in Magnetic Resonance Images. Proc. SPIE 2359:160-169, 1994.

14. R.Malladi, J.A.Sethian and B.C.Vemuri. Shape modeling with front propagation: a level set approach, IEEE Trans. on Pattern Analysis and Machine Intelligence, 17(2):158-174, Feb, 1995.

15. R.Malladi, R.Kimmel, D.Adalsteinsson, G.Sapiro, V.Caselles and J.A. Sethian. A geometric approach to segmentation and analysis of $3 \mathrm{D}$ medical images. Proc. $M M B I A, 1996$.

16. R.T.Schultz and A.Chakraborty. Magnetic resonance image analysis. In E. Bigler (Ed.), Handbook of Human Brain Function: Neuroimaging, pp:9-51. New York: Plenum Press, 1996.

17. L.D.Selemon, G.Rajkowska and P.S.Goldman-Rakic. Abnormally High Neuronal Density In The Schizophremic Cortex, A Morphometric Analysis Of Prefrontal Area 9 and Occipital Area 17. Arch Gen Psychiatry, vol52:pp805-828, Oct 1995.

18. J.A.Sethian. Level set methods:evolving interfaces in geometry, fluid mechanics, computer vision and materials science. Cambridge University Press, 1996.

19. P.C.Teo, G.Sapiro and B.A.Wandell. Creating connected representations of cortical gray matter for functional MRI visualization. IEEE Trans.Med. Imag., 16(6):852$863,1997$.

20. X.Zeng, L.H.Staib, R.T.Schultz and J.S.Duncan. Volumetric layer segmentation using coupled surfaces propagation. Proc. IEEE Conf. on Computer Vision and Pattern Recognition, pp:708-715, Santa Barbara, California, June, 1998. 